Temporal lobe epilepsy began at 2 to 6 months after the febrile seizure, at ages 7 mos to 3.5 years. Intracranial volume ipsilateral to the HS was relatively small in 2 of 3 affected twins, when compared to the unaffected twin. HS was not caused by perinatal abnormalities and was unrelated to birth order. The absence of HS in the unaffected twin is evidence against a genetic basis for HS. An acquired lesion secondary to prolonged febrile seizures is the more likely mechanism. (Jackson GD, McIntosh AM, Briellmann RS, Berkovic SF. Hippocampal sclerosis studied in identical twins. Neurology July 1998;51:78-84). (Reprints: Dr Graeme Jackson, Director, Centre for Brain Imaging Research, Austin and Repatriation Medical Centre, Heidelberg (Melbourne), Victoria 3084, Australia).

COMMENT. Monozygotic twin studies support an acquired basis for the hippocampal sclerosis associated with temporal lobe epilepsy, secondary to prolonged febrile seizures in early childhood. All three MZ pairs for which the proband had temporal lobe epilepsy (TLE) and HS were discordant for the clinical diagnosis of TLE. Definitive dysplastic changes were not uncovered by MR, but subtle changes could not be ruled out.

\title{
FEBRILE CONVULSIONS AND CONGENITAL HYPOTHYROIDISM
}

The incidence of febrile convulsions (FCs) among 63 children with congenital hypothyroidism $(\mathrm{CH})$ was compared to that of control children and patient's siblings in a study at Niigata University, Japan. Patients had been treated with L-thyroxine from 1 month of age. FCs had occurred in only one child with $\mathrm{CH}$ (1.6\%) compared to $8.2 \%$ of control children, $9.5 \%$ of the patient's siblings, and $6.4 \%$ of the patient's parents. (Asami T, Sasagawa F, Kyo S, Asami K, Uchiyama M. Incidence of febrile convulsions in children with congenital hypothyroidism. Acta Paediatr June 1998;87:623-626) (Respond: Dt T Asami, Department of Pediatrics, School of Medicine, Niigata University, Asahimachi-dori 1-757, Niigata, Japan).

COMMENT. Children with congenital hypothyroidism who have been treated regularly with thyroid hormone are less prone to have febrile convulsions. A review of systemic electrolyte and neuroendocrine mechanisms of epilepsy (Millichap JG. In Basic Mechanisms of the Epilepsies. Jasper HH, Ward AA, Pope A (eds), Boston, Little Brown, 1969) found that Timiras PS and Woodbury DM conducted much of the early experimental work on thyroid imbalance and seizures. Timiras showed that thyroxine increased brain excitability in rats, and Woodbury found that an increased seizure threshold in thyroidectomized rats was lowered by giving thyroid hormone. These alterations in brain excitability were correlated with changes in brain electrolytes. Clinical studies have demonstrated that seizures that accompany myxoema coma respond to thyroid treatment. Further studies of the influence of thyroid function on childhood seizures are needed.

\section{KETOGENIC DIET COMPLICATIONS}

Serious adverse events are reported in five (10\%) of 52 children, aged 1.516 years, treated with the ketogenic diet (4:1 ratio/fat: carbohydrate) over a 22month period at the Montefiore Medical Center and Albert Einstein College of Medicine, Bronx, NY. The Johns Hopkins KD protocol was followed, and most patients were started at a 4:1 ratio. All patients had intractable epilepsy and had received at least 3 antiepileptic drugs. At diet initiation, 29 were receiving valproate (VPA), of whom 4 developed complications within one month. These included hypoproteinemia, lipemia, hemolytic anemia, and Fanconi's renal tubular acidosis. Two had severe abnormalities of liver function tests, one with a 
concomitant thrombocytopenia. (Ballaban-Gil K, Callahan C, O'Dell C, Pappo M, Moshe S, Shinnar S. Complications of the ketogenic diet. Epilepsia July 1998;39:744-748). (Reprints: Dr K Ballaban-Gil, Epilepsy Management Center, Montefiore Medical Center, 111 E 210th St, Bronx, NY 10467).

COMMENT. Using the Johns Hopkins protocol for the ketogenic diet (KD) treatment of childhood refractory epilepsy, serious complications may occur in $10 \%$ of patients, often within one month of initiating the diet in a $4: 1 /$ ketogenic: antiketogenic ratio. Personally, I have preferred the Mayo Clinic method, which does not usually require the patient's hospitalization nor an initial period of starvation. The ratio of ketogenic to antiketogenic foods is modified over a 4 day period, beginning with a 1.1:1 ratio on the first day, 1.6:1 on the 2 nd, 2.2:1 on the 3rd, and 2.8:1 on the 4th. A 4:1 ratio is rarely required to obtain ketosis and only in older children (Millichap JG. Nutrition, Diet, and Child's Behavior. Springfield, IL, Charles C Thomas, 1986). With this Mayo method of KD introduction, I have not encountered the adverse reactions reported with the Hopkins protocol. The concomitant use of valproate with the Hopkins ketogenic diet may also be a factor in the incidence of adverse events reported.

\section{BRAIN NEOPLASMS}

\section{PROGNOSIS OF MEDULLOBLASTOMA}

The prognostic role of clinical, pathological, and therapeutic factors in cases of medulloblastoma reported in the literature are reviewed at the University of Turin, Italy. Improvements in neurosurgical techniques and the addition of cranio-spinal axis radiotherapy and chemotherapy can account for a drop in operative mortality from $32 \%$ before the 1960 s to $10 \%$ after 1970 , and an increase in 5 -year survival rate from $2-11 \%$ to $50-70 \%$ in the same time periods. Local recurrence in the posterior fossa is the most frequent cause of failed treatment, and higher doses of irradiation ( $>50 \mathrm{~Gy}$ ) may prolong survival but have long-term side effects. Low-dose craniospinal radiotherapy plus adjuvant chemotherapy provides survival rates similar to those of high-dose irradiation, with less sequelae. Treatment is planned according to Chang's staging scheme, cases showing distant seeding receiving chemotherapy. The extent of surgical resection plays a controversial role in prognosis, but many surgeons favor a wide excision. The role of age at diagnosis is also uncertain, some authors concluding that 5 -year survival is lower in children under 5 years, while others have found a better prognosis for infants, younger than 2 years. The prognostic significance of the pathology and cell differentiation of the tumor has been studied extensively with conflicting results. Tumors with a high proliferative rate are more susceptible to apoptosis and respond best to radiation. Almost $50 \%$ of medulloblastomas analyzed genetically exhibit deletion of the short arm of chromosome 17, a finding linked to shortened survival in some studies. (Giordana MT, Schiffer P, Schiffer D. Prognostic factors in medulloblastoma. Child's Nerv Syst June 1998;14:256-262). (Respond: Dr MT Giordana, Division of Neurology, Dept of Neuroscience, University of Turin, Via Cherasco, 15, 1-10126 Turin, Italy).

COMMENT. The prognosis of medulloblastoma is generally poor, $50 \%$ having recurrence, with death resulting within 5 to 7 years after diagnosis. Relapses after 8 years freedom from recurrence are rare. Factors having a significant influence on survival include radiotherapy and metastases at the time of diagnosis. The extent of surgical excision may also be important. Ongoing 